\title{
Il testosterone intratesticolare è aumentato negli uomini con sindrome di Klinefelter e potrebbe non essere rilasciato nel circolo ematico a causa di un'alterazione della vascolarizzazione testicolare-uno studio preliminare
}

\author{
Fabio Lanfranco $^{1} \cdot$ Stefano Allasia $^{1}$
}

Pubblicato online: 14 luglio 2015

(C) Springer International Publishing AG 2015

Commento a:

Intratesticular testosterone is increased in men with Klinefelter syndrome and may not be released into the bloodstream owing to altered testicular vascularization-a preliminary report.

F. Tüttelmann, O.S. Damm, M. Luetjens, M. Baldi, M. Zitzmann, S. Kliesch, E. Nieschlag, J. Gromoll, J. Wistuba, M. Simoni.

Andrology (2014) 2:275-281

La sindrome di Klinefelter (SK) è la più comune causa di infertilità maschile e insufficienza testicolare su base genetica. Si ritiene che la progressiva riduzione dei livelli ematici di testosterone in questi pazienti sia attribuibile a un deficit funzionale delle cellule di Leydig. Esperimenti condotti su modelli murini, tuttavia, hanno rilevato concentrazioni intratesticolari di testosterone paragonabili ai controlli wild-type. Lo scopo principale di questo studio è stato quello di valutare l'assetto ormonale sierico, intra-testicolare e le caratteristiche istologiche dei testicoli in 50 soggetti azoospermici, di cui 11 con SK, 30 con sindrome a sole cellule di Sertoli (SCOS) e 9 con azoospermia ostruttiva (controlli).

I pazienti con $\mathrm{SK}$ hanno mostrato un volume testicolare significativamente minore $(8,4 \pm 4,5 \mathrm{ml} ; p<0,01)$ rispetto agli altri pazienti. Nei soggetti con SK le concentrazioni di FSH e LH sono risultate più elevate e quelle di testosterone totale più basse rispetto ai controlli; i livelli di SHBG erano simili nei tre gruppi. Il testosterone intra-testicolare (ITT) è risultato significativamente maggiore nei soggetti

F. Lanfranco

fabio.lanfranco@unito.it

1 Divisione di Endocrinologia, Diabetologia e Metabolismo, Dipartimento di Scienze Mediche, Università di Torino, Torino, Italia azoospermici con SK, mentre la concentrazione di SHBG intra-testicolare non mostrava sostanziali differenze tra i 3 gruppi. Il rapporto ITT/T sierico nei soggetti con SK era significativamente più elevato rispetto a quello osservato nei soggetti con SCOS e nei controlli. Il rapporto ITT/LH non mostrava invece differenze tra i 3 gruppi.

L'analisi istologica del tessuto testicolare ha evidenziato una significativa riduzione del diametro medio dei tubuli seminiferi nei pazienti con SK e SCOS rispetto ai controlli. Inoltre, i soggetti con SK presentavano un numero di cellule di Leydig quadruplicato rispetto ai controlli e raddoppiato rispetto ai pazienti con SCOS. Non è stato possibile, invece, valutare con precisione la vascolarizzazione testicolare dal materiale bioptico a disposizione. Per ovviare a questo problema gli autori hanno utilizzato eleganti modelli murini, evidenziando che topi con disgenesia gonadica (41, XXY) mostrano rispetto ai controlli sani (40, XY) una netta riduzione del letto vascolare testicolare.

In conclusione, questo studio ha dimostrato che le concentrazioni intra-testicolari di testosterone sono significativamente aumentate nei soggetti con SK, nonostante la presenza di livelli sierici costantemente ridotti. Tali risultati rappresentano un'ulteriore prova che i bassi livelli di testosterone sierico rilevati nella SK non dipendono da alterazioni della steroidogenesi testicolare. In presenza di valori testicolari di SHBG non aumentati, gli autori sostengono che una riduzione della vascolarizzazione locale possa rappresentare la causa principale dell' alterato rilascio in circolo e del conseguente accumulo testicolare del testosterone. 